\title{
Improving energy harvesting in excited Duffing harvester device using a delayed piezoelectric coupling
}

\author{
Z. Ghouli ${ }^{1}$, M. Hamdi ${ }^{2}$, M. Belhaq ${ }^{1}$ \\ ${ }^{1}$ Faculty of Sciences Ain Chock, University Hassan II-Casablanca, Morocco \\ ${ }^{2}$ FST-Al Hoceima, Mohamed First University Oujda, Al Hoceima, Morocco
}

\begin{abstract}
The present work examines the influence of time delay introduced in the piezoelectric circuit of an excited Duffing harvester device with hardening stiffness on the vibration and voltage amplitudes. Specifically, we seek to exploit a delayed electrical circuit of the harvester to enhance its performance. We consider the case of a monostable system and we use a perturbation technique to approximate the periodic response and the corresponding voltage amplitude near the principal resonance. It is shown that for appropriate values of delay amplitude, the energy harvesting performance is improved over a certain range of coupling parameters and excitation frequencies. Numerical simulation is conducted to support the analytical predictions.
\end{abstract}

\section{Introduction}

A monostable piezoelectric nonlinear energy harvester $(\mathrm{EH})$ device consisting of a Duffing-type harvester with hardening stiffness coupled to a delayed piezoelectric circuit is considered in this paper. The objective is to examines the influence of time delay in the piezoelectric circuit on the amplitude of the response and the voltage amplitude.

Recently, a delayed Duffing-type monostable harvester subject to a harmonic excitation and coupled to a piezoelectric circuit was studied in the case where the time delay is introduced only in the mechanical subsystem [1]. The optimal performance of the harvester device in term of time delay parameters was investigated and it was shown that the induced large-amplitude quasiperiodic vibrations can be used to extract energy over broadband of excitation frequencies away from the resonance.

Here we examine the influence of time delay on $\mathrm{EH}$ in the case where the time delay is introduced in the electric circuit. To show the contribution of introducing time delay in the electrical circuit in impoving EH performance, a comparison is carried out with the case where time delay is absent [2].

In the next section we present the harvester system and we derive approximation of the periodic response and the voltage amplitude using the multiple scales method [3]. In Section 3 the influence of different system parameters of the harvester device on the EH performance is analyzed in the presence and absence of time delay. A summary of the results is given in the concluding section.

\section{Harvester system, vibration response and voltage amplitude}

Consider an excited Duffing oscillator coupled to an electrical circuit through a delayed piezoelectric device as shown in the schematic presented in Fig. 1.

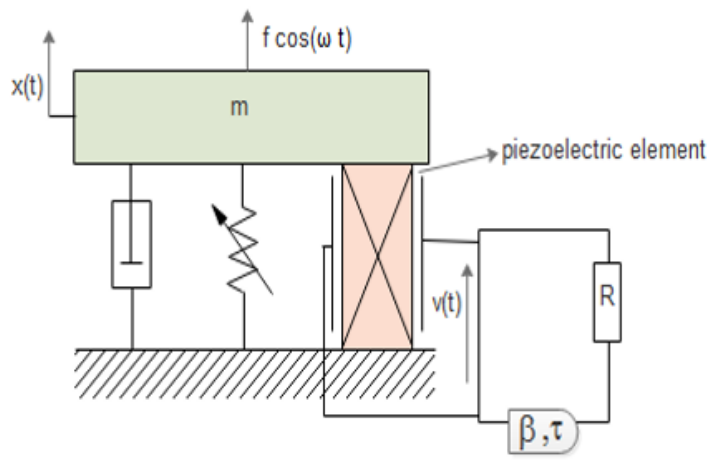

Fig. 1. Schematic description of the EH system

The governing equations for this harvester can be written in the dimensionless form as

$$
\begin{gathered}
\ddot{x}(t)+2 \mu \omega_{n} \dot{x}(t)+\omega_{n}^{2} x(t)+\alpha x(t)^{3}-\chi v(t)=f \cos (\omega t) \\
\dot{v}(t)+\lambda v(t)+\kappa \dot{x}(t)=\beta v(t-\tau)
\end{gathered}
$$

where $x(t)$ is the relative displacement of the rigid mass $m$, $v(t)$ is the voltage across the load resistance, $\omega_{n}$ is the natural frequency of the system, $\mu$ is the mechanical damping ratio, $\alpha$ is the stiffness parameter, $\chi$ is the piezoelectric coupling term in the mechanical attachment, $\kappa$ is the piezoelectric coupling term in the electrical circuit, $\lambda$ is the reciprocal of the time constant of the electrical circuit, $\beta$ and $\tau$ are, respectively, the feedback gain and time delay in the electrical circuit, and $f, \omega$ are, respectively, the amplitude and the frequency of the excitation. To investigate the influence of time delay in the electric circuit on the performance of the harvester, we approximate the response of the system near the primary resonance by introducing the resonance condition $\omega=\omega_{n}+\sigma$ where $\sigma$ is a detuning parameter.

The method of multiple scales [3] is implemented by introducing a bookkeeping parameter $\epsilon$ and scalling parameters as $\mu=\epsilon \tilde{\mu}, \alpha=\epsilon \tilde{\alpha}, \chi=\epsilon \tilde{\chi}, \sigma=\epsilon \tilde{\sigma}, f=\epsilon \tilde{f}$. 
Thus, Eqs. (1) and (2) take the form

$$
\begin{gathered}
\ddot{x}(t)+\omega_{n}^{2} x(t)=\epsilon\left[-2 \tilde{\mu} \omega_{n} \dot{x}(t)-\tilde{\alpha} x(t)^{3}+\tilde{\chi} v(t)+\tilde{f} \cos (\omega t)\right] \\
\dot{v}(t)+\lambda v(t)+\kappa \dot{x}(t)=\beta v(t-\tau)
\end{gathered}
$$

A solution to Eqs. (3) and (4) is given by

$$
\begin{aligned}
& x(t)=x_{0}\left(T_{0}, T_{1}\right)+\epsilon x_{1}\left(T_{0}, T_{1}\right)+O\left(\epsilon^{2}\right) \\
& v(t)=v_{0}\left(T_{0}, T_{1}\right)+\epsilon v_{1}\left(T_{0}, T_{1}\right)+O\left(\epsilon^{2}\right)
\end{aligned}
$$

where $T_{0}=t$, and $T_{1}=\epsilon t$. In terms of the variables $T_{i}$, the time derivatives become $\frac{d}{d t}=D_{0}+\epsilon D_{1}+O\left(\epsilon^{2}\right)$ and $\frac{d^{2}}{d t^{2}}=$ $D_{0}^{2}+\epsilon^{2} D_{1}^{2}+2 \epsilon D_{0} D_{1}+O\left(\epsilon^{2}\right)$ where $D_{i}^{j}=\frac{\partial^{j}}{\partial^{j} T_{i}}$. Substituting $(5)$ and (6) into (3) and (4) and equating coefficient of like powers of $\epsilon$, we obtain up to the second order the systems

$$
\begin{gathered}
D_{0}^{2} x_{0}+\omega_{n}^{2} x_{0}=0 \\
D_{0} v_{0}+\lambda v_{0}+\kappa D_{0} x_{0}=\beta v_{0 \tau} \\
D_{0}^{2} x_{1}+\omega_{n}^{2} x_{1}=-2 D_{0} D_{1} x_{0}-2 \tilde{\mu} \omega_{n} D_{0} x_{0}-\tilde{\alpha} x_{0}^{3}+\tilde{\chi} v_{0} \\
+\tilde{f} \cos \left(\omega_{n} T_{0}+\tilde{\sigma} T_{1}\right) \\
D_{0} v_{1}+\lambda v_{1}=-D_{1} v_{0}-\kappa D_{0} x_{1}-\kappa D_{1} x_{0}+\beta v_{1 \tau}
\end{gathered}
$$

Up to the first order the solution reads

$$
x_{0}\left(T_{0}, T_{1}\right)=A\left(T_{1}\right) e^{i \omega_{n} T_{0}}+\bar{A}\left(T_{1}\right) e^{-i \omega_{n} T_{0}}
$$

$v_{0}\left(T_{0}, T_{1}\right)=\frac{-\kappa i \omega_{n} A\left(T_{1}\right)}{\lambda+i \omega_{n}-\beta e^{-i \omega_{n} \tau}} e^{i \omega_{n} T_{0}}+\frac{\kappa i \omega_{n} \bar{A}\left(T_{1}\right)}{\lambda-i \omega_{n}-\beta e^{i \omega_{n} \tau}} e^{-i \omega_{n} T_{0}}$

where $A\left(T_{1}\right)$ and $\bar{A}\left(T_{1}\right)$ are unknown complex conjugate functions. Substituting Eqs. (11) and (12) into (9) and (10) and eliminating the secular terms, one obtains

$$
-2 i \omega_{n}\left(D_{1} A\right)-2 i \tilde{\mu} \omega_{n}^{2} A-3 \tilde{\alpha} A^{2} \bar{A}-\frac{\kappa i \omega_{n} \tilde{\chi} A}{\lambda+i \omega_{n}-\beta e^{-i \omega_{n} \tau}}+\frac{\tilde{f}}{2} e^{i \tilde{\sigma} T_{1}}=0
$$

Expressing $A=\frac{1}{2} a e^{i \theta}$ where $a$ and $\theta$ are the amplitude and the phase of the modulation, we obtain up to the first order the modulation equations

$$
\left\{\begin{array}{l}
\frac{d a}{d t}=S_{1} a+S_{4} \sin (\gamma) \\
a \frac{d \gamma}{d t}=S_{2} a+S_{3} a^{3}+S_{4} \cos (\gamma)
\end{array}\right.
$$

where $S_{i}(i=1, \ldots, 4)$ are given in Appendix and $\gamma=\tilde{\sigma} T_{1}-$ $\theta$. The steady-state response of system (14), corresponding to periodic oscillations of Eqs. (3) and (4), are determined by setting $\frac{d a}{d t}=\frac{d \gamma}{d t}=0$. Eliminating the phase, we obtain the following sixth-order algebraic equation in $a$

$$
\left(S_{1} a\right)^{2}+\left(S_{2} a+S_{3} a^{3}\right)^{2}=S_{4}^{2}
$$

which can $b$ written in the form

$$
B a^{6}+C a^{4}+D a^{2}+E=0
$$

where $B=S_{3}^{2}, C=2 S_{2} S_{3}, D=S_{1}^{2}+S_{2}^{2}$ and $E=-S_{4}^{2}$. The discriminant of Eq. (16) reads

$$
\Delta=\frac{P^{3}}{27}+\frac{Q^{2}}{4}
$$

where $P=\frac{D}{B}-\frac{C^{2}}{3 B^{2}}$ and $Q=\frac{2}{27}\left(\frac{C}{B}\right)^{3}-\frac{C D}{3 B^{2}}+\frac{E}{B}$. Equation (16) has three real positive roots if $\Delta$ is negative and one positive root if $\Delta$ is positive.

The solution given by Eqs. (11) and (12) can be written as $x_{0}\left(T_{0}, T_{1}\right)=a \cos \left(\omega_{n} t+\theta\right)$ and $v_{0}\left(T_{0}, T_{1}\right)=V \cos \left(\omega_{n} t+\right.$ $\left.\theta+\arctan \frac{\lambda-\beta \cos \left(\omega_{n} \tau\right)}{\omega_{n}+\beta \sin \left(\omega_{n} \tau\right)}\right)$ such that the condition $\omega_{n}+\beta \sin \left(\omega_{n} \tau\right)$ $\neq 0$ is satisfied. Moreover, the voltage amplitude $V$ is given by

$$
V=\frac{\kappa \omega_{n}}{\sqrt{\left(\lambda-\beta \cos \left(\omega_{n} \tau\right)\right)^{2}+\left(\omega_{n}+\beta \sin \left(\omega_{n} \tau\right)\right)^{2}}} a
$$

\section{Main results}

Next, the influence of different parameters of the system on vibration and voltage amplitudes is examined using Eqs. (15) and (18), respectively.

Figures $2 \mathrm{a}$ and $2 \mathrm{~b}$ show, respectively, the variation of the amplitude of the periodic vibrations and the voltage $V$ versus $\sigma$ for the excitation amplitude $f=0.1$. The undelayed case $(\beta=0)$ is presented by a grey line and the delayed one $(\beta \neq 0)$ is indicated by a black line. The analytical prediction (solid lines) is compared to results obtained by numerical simulation using dde23 algorithm [4] for the delayed case and the method of Runge Kutta of order 4 for the undelayed case. It can be observed from the figures that the EH performance is improved by introducing the time delay in the electric circuit, especially near the resonance peak.

Figure 3 shows the variation of the amplitude of the periodic response and the voltage amplitude $V$ versus $\sigma$ for larger value of the forcing amplitude $f=1.5$. The grey line corresponds to the undelayed case $(\beta=0)$ and the black one is obtained in the delayed one. As in the previous case (Fig. 2 for $f=0.1$ ) the voltage amplitude is increased around the resonance peak in the case of delayed circuit.

Similarly, the variation of the amplitude of vibration and voltage versus the piezoelectric coupling term in the electrical circuit $\kappa$ are depicted in Fig. 4 showing the range of parameter $\kappa$ where a substantial EH performance is achieved (Fig. 4b). The variation of the amplitudes of vibration and voltage as a function of the piezoelectric coupling term in the mechanical attachment $\chi$ are shown in Fig. 5 for $\beta=0$ (undelayed circuit, grey line) and $\beta=\lambda$ (delayed circuit, black line). Inspection of Fig. 5 shows that for values of $\sigma$ taken relatively away from the resonance, the range of $\chi$ where a better performance of EH is obtained (Fig. 5b).

\section{Conclusion}

We have studied the EH performance in a monostable Duffing forced oscillator with hardening stiffness coupled to a delayed piezoelectric harvester device. The analysis was carried out near the primary resonance and a perturbation method was performed to obtain approximation of vibration and voltage amplitudes. The influence of time delay introduced in the piezoelectric subsystem on the EH performance of the harvester was studied. Results showed that 


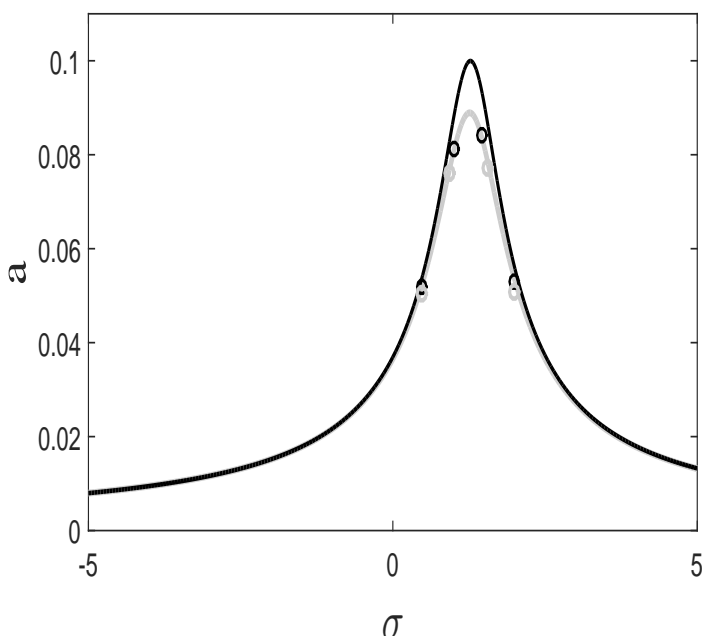

(a)

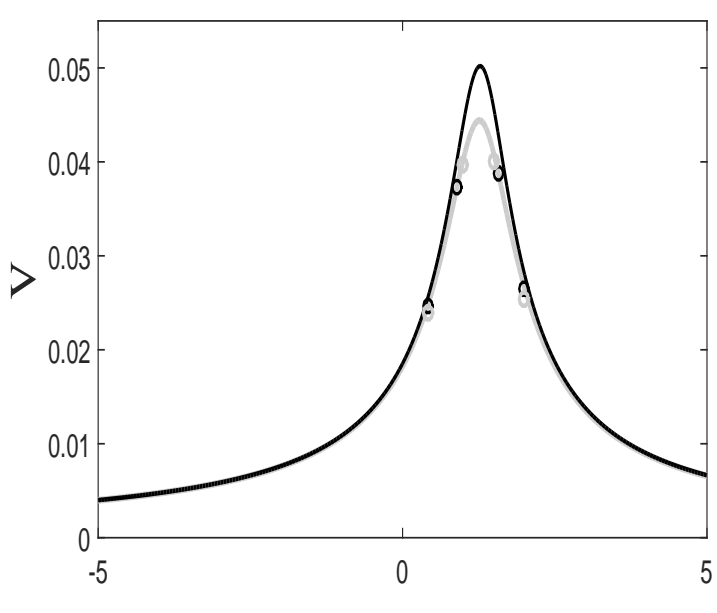

(b)

$\sigma$

Fig. 2. Vibration (a) and voltage (b) amplitudes vs $\sigma$ for $f=$ $0.1, \chi=0.05, \kappa=0.5, \omega_{n}=1, \mu=0.5, \alpha=5, \lambda=0.05$, and $\tau=6.2$. Black (grey) line for delayed (undelayed) electric circuit, $\beta=\lambda(\beta=0)$. Analytical prediction (solid lines); numerical simulation (black (grey) circles for delayed (undelayed) electric circuit).

in the presence of time delay in the electrical circuit the voltage amplitude increases at certain range of coupling parameters especially in the vicinity of the resonance peak. In other words, by tuning the delay amplitude in the electric circuit, it is possible to optimize the power output performance over certain range of the piezoelectric coupling terms.

\section{Appendix}

$S_{1}=-\mu \omega_{n}-\frac{\chi^{\kappa}\left(\lambda-\beta \cos \left(\omega_{n} \tau\right)\right)}{2\left[\left(\lambda-\beta \cos \left(\omega_{n} \tau\right)\right)^{2}+\left(\omega_{n}+\beta \sin \left(\omega_{n} \tau\right)\right)^{2}\right]}$

$$
S_{2}=\sigma-\frac{\chi \kappa\left(\omega_{n}+\beta \sin \left(\omega_{n} \tau\right)\right)}{2\left[\left(\lambda-\beta \cos \left(\omega_{n} \tau\right)\right)^{2}+\left(\omega_{n}+\beta \sin \left(\omega_{n} \tau\right)\right)^{2}\right]}
$$

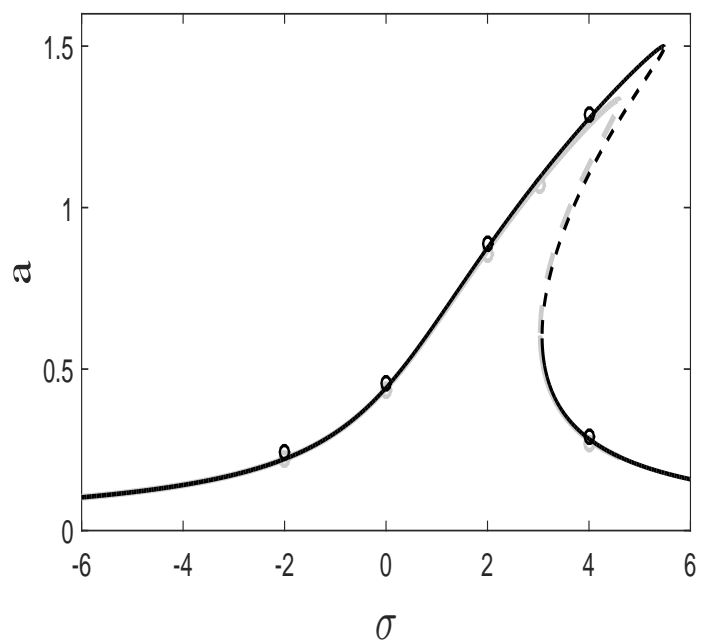

(a)

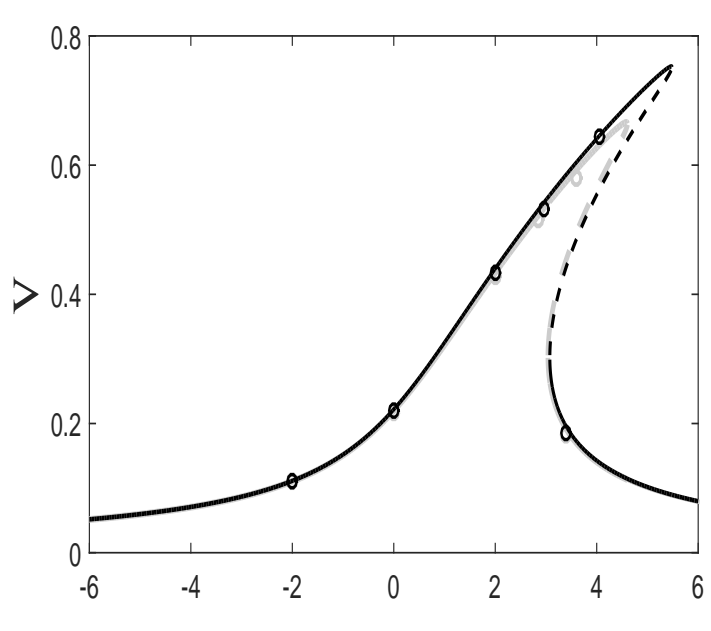

(b)

$\sigma$

Fig. 3. Vibration (a) and voltage (b) amplitudes vs $\sigma$ for $f=$ $1.5, \chi=0.05, \kappa=0.5, \omega_{n}=1, \mu=0.5, \alpha=5, \lambda=0.05$, and $\tau=6.2$. Black (grey) line for delayed (undelayed) electric circuit $\beta=\lambda(\beta=0)$. Analytical prediction (solid lines for stable and dashed line for unstable) and numerical simulation (Black (grey) circles for delayed (undelayed) electric circuit).

$$
\begin{gathered}
S_{3}=-\frac{3 \alpha}{8 \omega_{n}} \\
S_{4}=\frac{f}{2 \omega_{n}}
\end{gathered}
$$

\section{References}

1. Z. Ghouli, M. Hamdi, F. Lakrad, M. Belhaq, Quasiperiodic energy harvesting in a forced and delayed Duffing harvester device, J. Sound Vib. 407 (2017) 271-285.

2. A. Erturk, D.J. Inman, Piezoelectric Energy Harvesting, John Wiley, 2011.

3. Nayfeh, A.H., Mook, D.T.: Nonlinear Oscillations. Wiley, New York, 1979. 


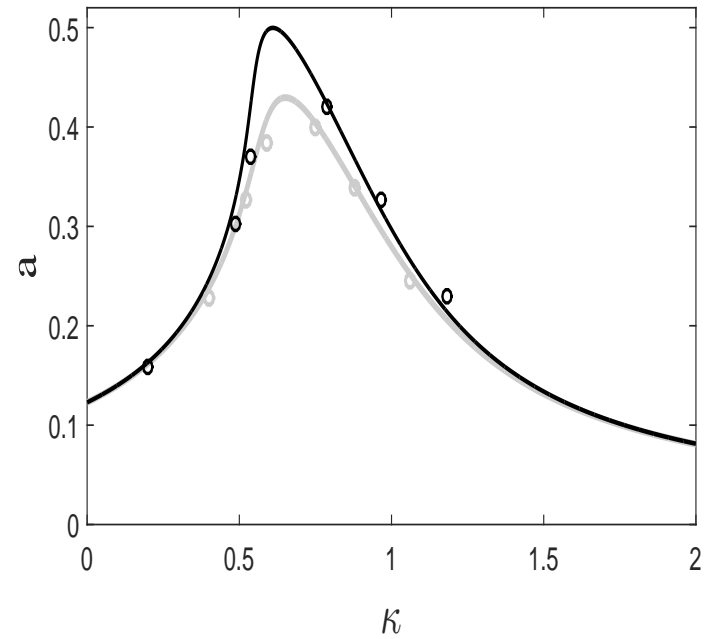

(a)

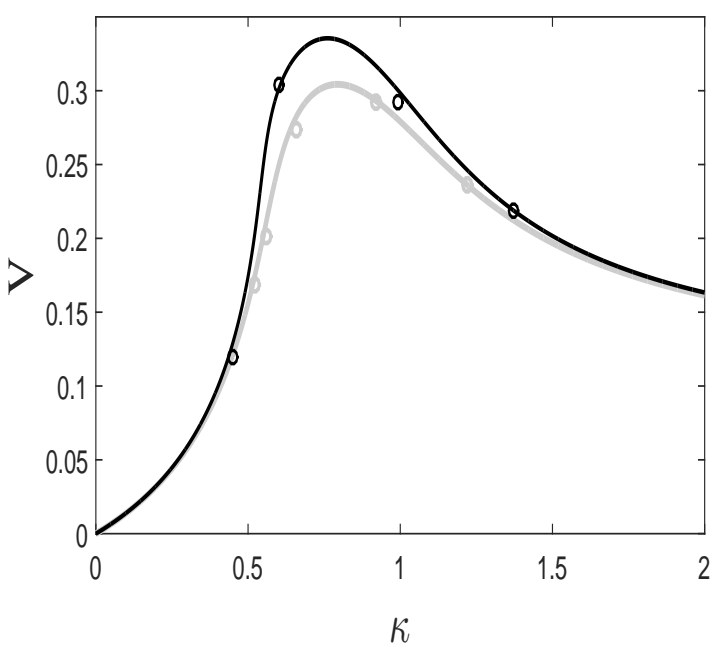

(b)

Fig. 4. Vibration (a) and voltage (b) amplitudes vs $\kappa$ for $\sigma=$ $2, \chi=5, f=0.5, \omega_{n}=1, \mu=0.5, \alpha=5, \lambda=0.05$, and $\tau=6.2$. Black (grey) line for delayed (undelayed) electric circuit $\beta=\lambda$ $(\beta=0)$. Analytical prediction (solid lines) and numerical simulation (Black (grey) circles for delayed (undelayed) electric circuit).

4. L.F. Shampine, S. Thompson, Solving delay differential equations with dde23. PDF available on-line at http: //www.radford.edu/ thompson/webddes/tutorial.pdf (2000).
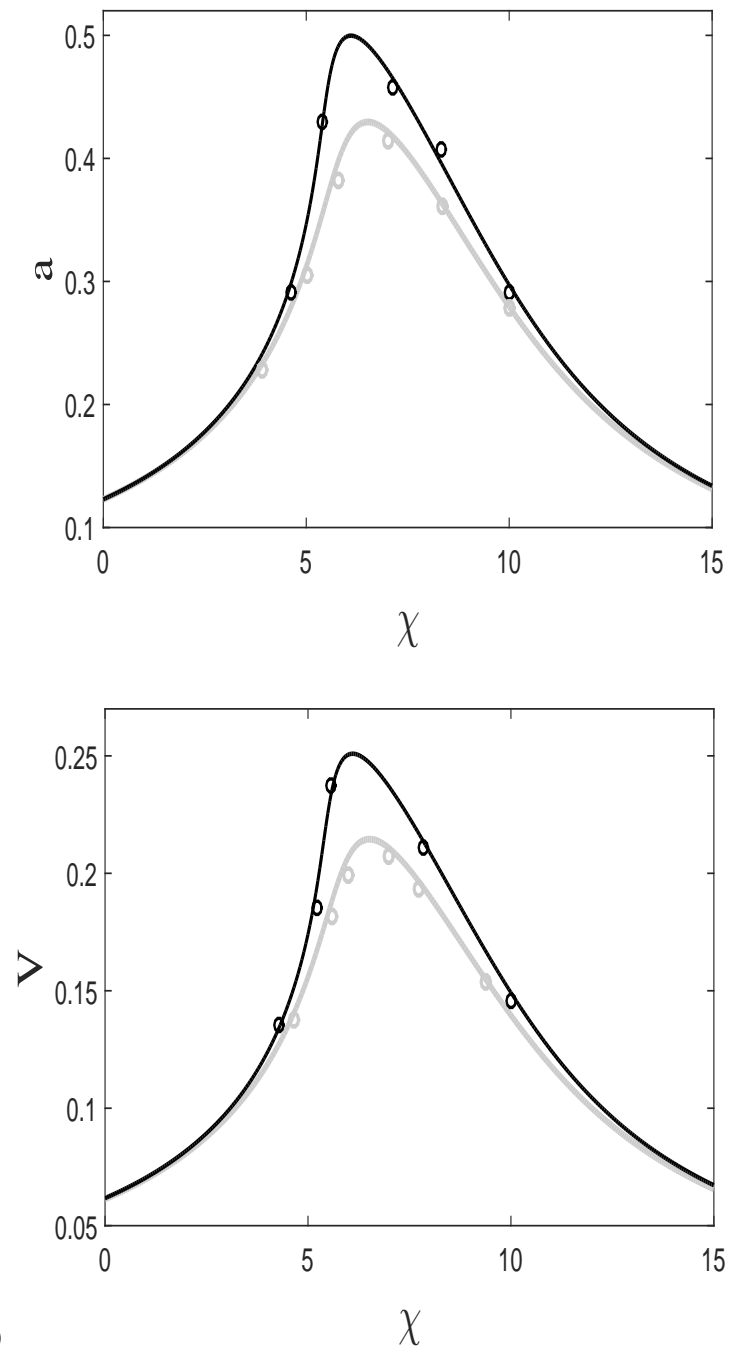

Fig. 5. Vibration (a) and voltage (b) amplitudes vs $\chi$ for $\sigma=$ $2, \kappa=0.5, f=0.5, \omega_{n}=1, \mu=0.5, \alpha=5, \lambda=0.05$, and $\tau=6.2$. Black (grey) line for delayed (undelayed) electric circuit $\beta=\lambda$ $(\beta=0)$. Analytical prediction (solid lines) and numerical simulation (Black (grey) circles for delayed (undelayed) electric circuit). 